\title{
Grain-size characteristics of linear dunes on the northern margin of Qarhan Salt Lake, northwestern China
}

\author{
LI Jiyan*, DONG Zhibao, ZHANG Zhengcai, QIAN Guangqiang, LUO Wanyin, LU Junfeng
}

Key Laboratory of Desert and Desertification, Cold and Arid Regions Environmental and Engineering Research Institute, Chinese Academy of Sciences, Lanzhou 730000, China

\begin{abstract}
In order to clarify the formation mechanism of linear dunes on the northern margin of Qarhan Salt Lake, northwestern China, we analyzed the grain-size and sorting parameters of the dune and interdune sands. The surface sands $(0-30 \mathrm{~mm})$ from the dune base to the crest of both flanks and interdune corridors were sampled along transects from upwind to downwind through the dune field. The results indicated that the grain-size distribution differed at different positions between and within the dunes. The frequency curve for dune sands mainly showed a bimodal distribution, while the interdune sediments showed a trimodal distribution. The grain size distribution of the linear dunes showed a finer crest pattern, i.e. the crests were composed of sands that were generally finer, better sorted than those of base sands. In addition, at the dune field scale, the dune crest sands were tending to become much finer but sorting became worse along the downwind transects. However, the grain-size parameters of sediments in the interdune corridors showed no clear pattern. The results demonstrated that the grain size and sorting parameters exhibited a systematic change not only at the individual scale but also at the dune field scale. Our results quantitatively estimate the limited role of cohesive sediments on the formation of linear dune under unidirectional wind regime. More attention should be paid to a long-term wind regime observation, internal sedimentary structures and their formation ages.
\end{abstract}

Keywords: linear dune; longitudinal dune; grain-size; parameters; Qarhan Salt Lake; Qaidam Basin

Citation: LI Jiyan, DONG Zhibao, ZHANG Zhengcai, QIAN Guangqiang, LUO Wanyin, LU Junfeng. 2015. Grain-size characteristics of linear dunes on the northern margin of Qarhan Salt Lake, northwestern China. Journal of Arid Land, 7(4): 438-449. doi: 10.1007/s40333-015-0005-4

Linear (longitudinal) dunes are widely distributed in Australia, Africa, Arabian Peninsula and parts of Central Asia, as well as in the deserts of North and South America (Lancaster, 1982; Tsoar, 1989; Qian et al., 2009). However, they are found not only on the Earth, but also on the Mars (Edgett and Blumberg, 1994; Lee and Thomas, 1995) and the Titan (Lancaster, 2006; Lorenz et al., 2006). This potentially makes them the most widespread aeolian landform in the solar system. The extensive distribution of linear dunes around the world has resulted in various local names for them, such as "long ridges" in Australia and "seif dunes" in Arab countries. The term "linear" emphasizes the morphology of the dunes, whereas "longitudinal" emphasizes their genesis (Rubin and Hesp, 2009). Linear dunes are characterized by their considerable length, straightness, parallelism and regular inter-crest spacing (Lancaster, 1995). Despite their wide occurrence, the mechanism responsible for the formation of linear dunes is still debated.

Generally, linear dunes are formed under two environments: one is that bidirectional winds blow across loose and soft sediments and the other is that unidirectional air currents blow over sediments that are locally stabilized by upwind obstacles, vegetation and cohesive sediments (Rubin and Hesp, 2009). In the former

*Corresponding author: LI Jiyan (E-mail: jyli@1zb.ac.cn)

Received 2014-10-06; revised 2015-01-16; accepted 2015-01-22

(C) Xinjiang Institute of Ecology and Geography, Chinese Academy of Sciences, Science Press and Springer-Verlag Berlin Heidelberg 2015 
situation, wind regime plays an important role. However, in the latter, sediment characteristics appear to be crucial. Linear dunes that formed in the leeward of fixed obstacles or that have been stabilized by vegetation have been studied systematically for decades on Earth (Bagnold, 1941; Tsoar, 1989; Herrmann et al., 2008) and more recent years on Titan (Rubin and Hesp, 2009). However, there have been few studies on the role of cohesive sediments in the formation of linear dunes. By spraying crude oil in the presence of a continuous sand supply, Kerr and Nigra (1952) observed the transformation of a barchan dune into an elliptical dome-shaped dune as a result of the increased sediment cohesiveness. By considering the similarity of Martian dunes to the behavior of these oil-soaked barchans, Herrmann et al. (2008) hypothesized that linear dunes at the north pole of Mars formed under the influence of unidirectional winds blowing over sediments that became cohesive by the presence of ice, frozen carbon dioxide and mineral salts. Rubin and Hesp (2009) provided field evidence of transverse dunes transforming into linear dunes in the Qaidam Basin in response to increasing sediment cohesiveness.

The content of silt and clay is a key factor in the cohesiveness of dune sediments, and this means that understanding the grain-size characteristics of sediments is essential to understanding the formation of linear dunes when unidirectional winds blow over cohesive sediments. In addition, by determining the threshold velocity of sediments, the grain-size distribution controls the effects of the wind regime on dune fields and on the dominant strike direction of the dune field (Qian et al., 2012). The observations of Warren (1972) suggested that the distinct morphologies of dunes may be related to their grain-size characteristics.

Three overall categories of grain-size patterns have been described for linear dunes: dunes with a finer crest (i.e. with enrichment in fine particles compared with the dune's base), dunes with a coarser crest and dunes with no difference (Lancaster, 1981; Livingstone and Warren, 1996). The latter pattern is rare. Warren (1971) reported no differences in grain-size and sorting parameters for linear dunes in the central Ténéré Desert. Buckley (1989) and Livingstone et al. (1999) found that linear dunes in both central Austral- ia and southwestern Kalahari showed a slight variation of grain-size. Linear dunes in the Namib Desert are typical examples of the pattern with a finer crest (Lancaster, 1981; Watson, 1986; Livingstone, 1987). Although the grain-size range of these linear dunes is small, the sand at the crest is generally finer, better sorted and less skewed towards the coarse side of the distribution than sands on the slopes and at the base of the dune (Livingstone and Warren, 1996). These characteristics can be attributed to the aeolian sorting processes that operate on the dune (Lancaster, 1981). In contrast, the studies of Folk (1971) in the Simpson Desert, and of Lancaster (1986) and Thomas (1988) in the Kalahari, all indicated a pattern with coarser, but better sorted on the crest sands than that of the slopes and interdune corridors. This pattern can be explained by the nature of the sediments in the source area: if the mean grain-size of the source is finer than that of optimum saltation load, then a coarser crest pattern would develop, and vice versa.

The linear dunes in China's Qaidam Basin were described by Hesp et al. (1989), whose investigation corroborated the lateral migration of linear dunes with field evidence. With the discovery of linear dunes on Mars (Edgett and Blumberg, 1994; Lee and Thomas, 1995) and Titan (Lancaster, 2006; Lorenz et al., 2006), the mechanism responsible for the formation of the Chinese linear dunes was used to extrapolate the mechanisms on other worlds, but the actual cause is still being debated (Rubin and Hesp, 2009; Zhou et al., 2012). When the Qinghai-Tibetan Railway was constructed, Qian and Liu (1994) investigated the development of aeolian disasters that resulted from wind erosion of sediments in this area, and they analyzed the grain-size characteristics of these linear dunes. However, given the high silt and clay contents and the cohesiveness of the dune sediments, their method is likely to underestimate the content of fine particles compared with other methods such as sedimentation in water. Another problem is that previous studies have generally used limited sediment samples to extrapolate the grain-size characteristics of a whole field of linear dunes (Lancaster, 1981; Watson, 1986).

In the present study, we systematically collected samples of surface sediments in a series of dune and interdune profiles at many locations along the direction in which the linear dunes were extending. Our 
aim was to demonstrate and quantify the variation in grain-size and sorting parameters for individual dunes and interdune areas and at the scale of the whole dune field.

\section{Study area and methods}

Qarhan Salt Lake is located in the center of the southern Qaidam Basin of northwestern China (Fig. 1). Because it developed at the lowest point, it gathered water from throughout the basin, leading to the deposition of thick lacustrine and fluvial sediments. It is currently composed of a few isolated perennial and seasonal brine lakes and an extensive dry playa bed. The lake lies at an elevation of 2,675 m, in a hyper-arid region where annual precipitation averages less than $30 \mathrm{~mm}$ and potential evaporation averages more than $3,000 \mathrm{~mm}$. The local wind regime is dominated by a narrowly unimodal wind regime, with wind predominantly from west to northwest. The resultant sand transport direction is along an azimuth of $124^{\circ}$ (Zhou et al., 2012). The combination of thick sediment deposits and strong winds led to the formation of linear dunes.

The linear dune field parallels the lake's shore and extends from WNW to ESE, with a length of nearly $40 \mathrm{~km}$ and a width of 4 to $6 \mathrm{~km}$ (Fig. 1a; Qian and Liu, 1994). The cross-section of these linear dunes is close to an isosceles triangle, with both flanks slightly concave in the middle. The slope angle at the bottom of
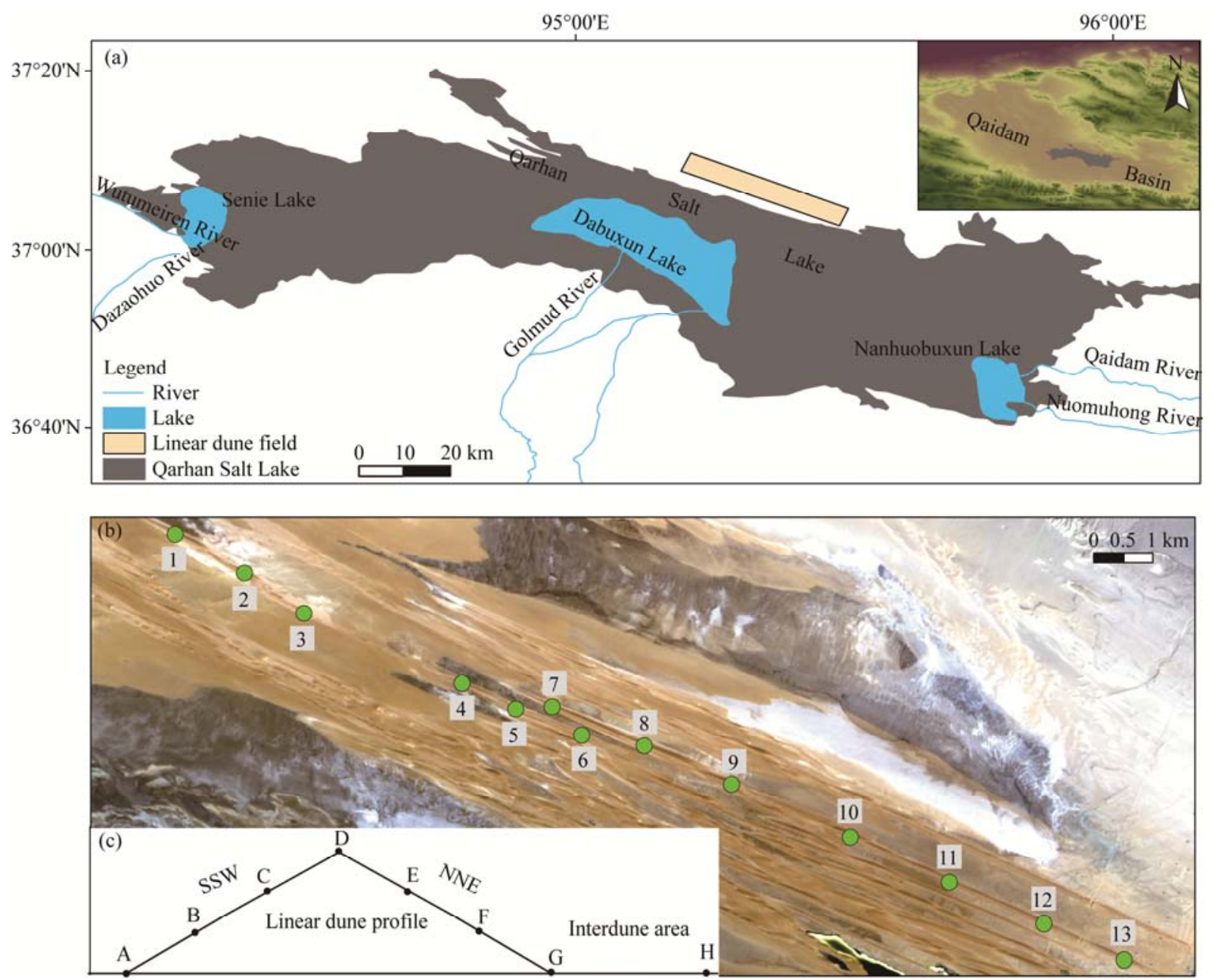

Fig. 1 The location of study area (a) and the distribution of the sampling sites (1 to 13) for the profiles in the linear dune field (b). Locations of the eight samples in each profile (c): A-C represent samples from the base, lower and upper parts of the dune's SSW flank, respectively; D represents the dune's crest; $E-G$ represent the upper, lower and base parts of the dune's NNE flank, respectively; and $H$ represents the interdune area about $80 \mathrm{~m}$ from the base of the dune's flank. 
the flank ranges from $5^{\circ}$ to $8^{\circ}$, gradually increases to $18^{\circ}-20^{\circ}$ in the middle, and reaches an angle of repose between $30^{\circ}$ and $36^{\circ}$ near the crest. The high content of soluble salts in the linear dune sediments explains the higher angle of repose at the dune's crest. Linear dunes in the dune field have stable strikes of about $115^{\circ}$, which is closely parallel to the resultant sand transport direction, with lengths of 4 to $12 \mathrm{~km}$, widths of 30 to $200 \mathrm{~m}$, heights of 5 to $30 \mathrm{~m}$ and a spacing of 100 to $600 \mathrm{~m}$ (Zhou et al., 2012).

For investigating the variation of grain-size and sorting parameters in the dune and interdune areas and at the scale of whole dune field, we established 13 profiles that extended from the base of one flank of the dune to the interdune area of the other flank, with the profiles covering a distance of nearly $20 \mathrm{~km}$ along the strike direction (Figs. $1 \mathrm{~b}$ and c). In each profile, we collected the surface sediments (to a depth of $30 \mathrm{~mm}$ ) at eight positions: seven on the dune and one in the interdune corridor (Fig. 1c). We collected a total of 104 surface sediment samples. We determined the grain-size distribution using a Malvern Mastersizer 2000 (Malvern Instruments, Malvern, UK). Based on the data, we calculated (in $\phi$ units) the mean grain-size $\left(\mathrm{M}_{\mathrm{Z}}\right)$, its standard deviation ( $\sigma_{\mathrm{I}}$, named sorting), the distribution's skewness $\left(\mathrm{SK}_{\mathrm{I}}\right)$ and kurtosis $\left(\mathrm{K}_{\mathrm{g}}\right)$, using the equations of Folk and Ward (1957).

\section{Results}

\subsection{Constituents of the sediments}

The physical constituents of the dune sediments reflect both the characteristics of sedimentary environment and the strength of the aeolian sorting processes that operated on the dune. The particle-size distributions based on the mean values from the 13 profiles indicate that the size distributions differed at different positions between and within the dunes. The bases of the two flanks (A and $G$ ) were mainly composed of medium to very coarse sands, whereas the upper parts, including the crest (B to F), were dominated by fine sands. The content of fine particles was slightly smaller on SSW flank than on NNE flank, and the content of coarse particles (especially coarse sand) was much higher at the base of SSW flank. The high content of fine sands ( 2 to $3 \phi$ values) at the crest, which is the optimum saltation load, indicates that aeolian sorting is much stronger at the dune's crest than at other positions (Fig. 2, Table 1). This can also be inferred by the fact that floating sands only occur at the dune's crest. Special attention should be paid to the high contents of clay (1.32\%) and silt (3.63\%) particles; their total of nearly $5 \%$ is far higher than the range $(0.63 \%$ to $4.60 \%)$ previously reported for other linear dune fields (Lancaster, 1981).

The interdune corridor is an important component of the dune field, and some researchers have proposed that it's the source area of the dune sediments (Folk, 1971). Compared with the dune's sediments, the sediments of the interdune corridors had a nearly symmetrical distribution dominated by fine sands (Fig. $2 \mathrm{H})$. The much higher content of clay (3.73\%) and silt $(12.34 \%)$ particles in the interdune corridors can be explained by the "protectionist theory" proposed by Warren (1972); that is, finer particles transported by saltation may penetrate the surface layer, and will subsequently be protected from the wind by coarse particles at the surface. In addition, there is a salt crust of approximately $20 \mathrm{~cm}$ thick. The content of coarse and very coarse sands in the interdune corridors was also much higher than that in the dune sediments sampled from the upper flanks and crest of the dune. The high contents of fine particles (clay and silt) and of coarse particles indicate poor sorting of sediments in the interdune corridor, which agrees with previous results for the other linear dune fields (Lancaster, 1981, 1986). Table 1 provides statistical details of the dune and interdune sediments.

\subsection{Frequency curves}

Figure 3 shows typical frequency curves for the grain-sizes for the sediments at different positions on the linear dune and in the interdune corridor. Except for the samples from the base of SSW flank (position A) and the interdune corridor sediments (position $\mathrm{H}$ ), the frequency curves show a clear bimodal distribution, with one main peak representing fine sand $(2.32$ to $2.82 \phi$ ) and a secondary peak representing silt (6 $\phi)$. The curve for the sediments at the base of NNE flank (position $\mathrm{G}$ ) also displays a bimodal distribution, but differs from the other bimodal distributions in a main peak for medium sands $(1.49 \phi)$ and a more 


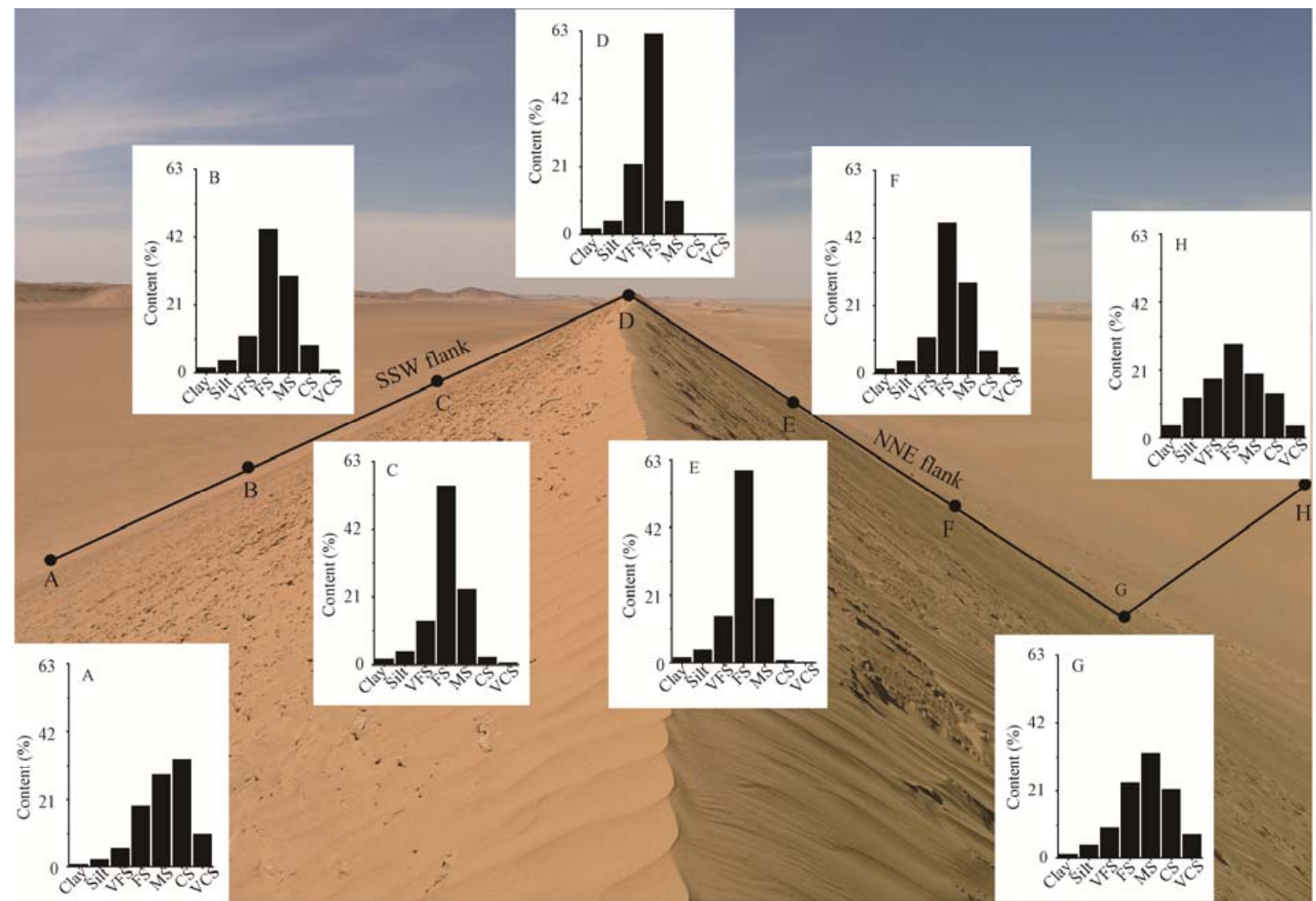

Fig. 2 Grain-size distributions of the samples from the linear dunes and interdune corridors. VFS, very fine sand; FS, fine sand; MS, medium sand; CS, coarse sand; VCS, very coarse sand.

Table 1 Ranges and mean values for the grain-size distribution at different positions on the dunes and in the interdune corridors

\begin{tabular}{|c|c|c|c|c|c|c|c|c|}
\hline \multirow{2}{*}{ Position } & & Clay & Silt & VFS & FS & MS & $\mathrm{CS}$ & VCS \\
\hline & & $<0.005 \mathrm{~mm}$ & $0.005-0.063 \mathrm{~mm}$ & $0.063-0.125 \mathrm{~mm}$ & $0.125-0.25 \mathrm{~mm}$ & $0.25-0.5 \mathrm{~mm}$ & $0.5-1 \mathrm{~mm}$ & $>1 \mathrm{~mm}$ \\
\hline \multirow{2}{*}{ A } & Range & $0.03-1.82$ & $0.83-4.40$ & $0.67-10.59$ & $0.43-73.41$ & $14.97-56.36$ & $0.00-55.92$ & $0.00-25.65$ \\
\hline & Mean & 0.65 & 2.11 & 5.70 & 19.10 & 28.60 & 33.51 & 10.33 \\
\hline \multirow{2}{*}{ B } & Range & $0.55-4.50$ & $2.07-8.54$ & $5.23-27.38$ & $19.47-69.08$ & $8.45-45.23$ & $0.00-29.00$ & $0.00-5.06$ \\
\hline & Mean & 1.43 & 3.69 & 11.36 & 44.25 & 30.02 & 8.50 & 0.75 \\
\hline \multirow{2}{*}{$\mathrm{C}$} & Range & $0.88-2.68$ & $2.99-5.07$ & $5.69-25.17$ & $35.37-68.50$ & $12.84-31.30$ & $0.00-12.57$ & $0.00-3.15$ \\
\hline & Mean & 1.55 & 3.85 & 13.54 & 55.32 & 23.22 & 2.11 & 0.41 \\
\hline \multirow{2}{*}{$\mathrm{D}$} & Range & $0.84-3.81$ & $2.91-5.16$ & $6.09-34.67$ & $50.68-70.72$ & $1.17-31.34$ & $0.00-1.53$ & $0.00-1.39$ \\
\hline & Mean & 1.69 & 4.03 & 21.60 & 61.98 & 10.45 & 0.14 & 0.11 \\
\hline \multirow{2}{*}{$\mathrm{E}$} & Range & $1.06-3.28$ & $2.85-5.38$ & $5.59-26.18$ & $43.03-72.58$ & $8.64-36.42$ & $0.00-6.57$ & $0.00-0.71$ \\
\hline & Mean & 1.49 & 3.91 & 14.40 & 59.58 & 19.96 & 0.60 & 0.05 \\
\hline \multirow{2}{*}{$\mathrm{F}$} & Range & $0.53-3.17$ & $2.43-6.97$ & $0.20-28.78$ & $9.11-62.77$ & $14.35-48.62$ & $0.00-43.63$ & $0.00-11.96$ \\
\hline & Mean & 1.29 & 3.80 & 11.30 & 46.63 & 28.15 & 7.11 & 1.71 \\
\hline \multirow{2}{*}{ G } & Range & $0.17-6.19$ & $0.82-20.54$ & $0.07-33.48$ & $5.48-38.98$ & $5.97-60.79$ & $2.50-48.07$ & $0.00-53.38$ \\
\hline & Mean & 1.14 & 4.03 & 9.70 & 23.47 & 32.69 & 21.35 & 7.62 \\
\hline \multirow{2}{*}{$\mathrm{H}$} & Range & $0.82-8.56$ & $2.37-39.69$ & $5.24-28.03$ & $13.95-42.49$ & $6.38-34.03$ & $3.07-36.47$ & $0.09-14.52$ \\
\hline & Mean & 3.73 & 12.34 & 18.06 & 28.99 & 19.61 & 13.68 & 3.59 \\
\hline
\end{tabular}

Note: A-H mean the sampling sites, shown in Fig. 1c. VFS, very fine sand; FS, fine sand; MS, medium sand; CS, coarse sand; VCS, very coarse sand. 

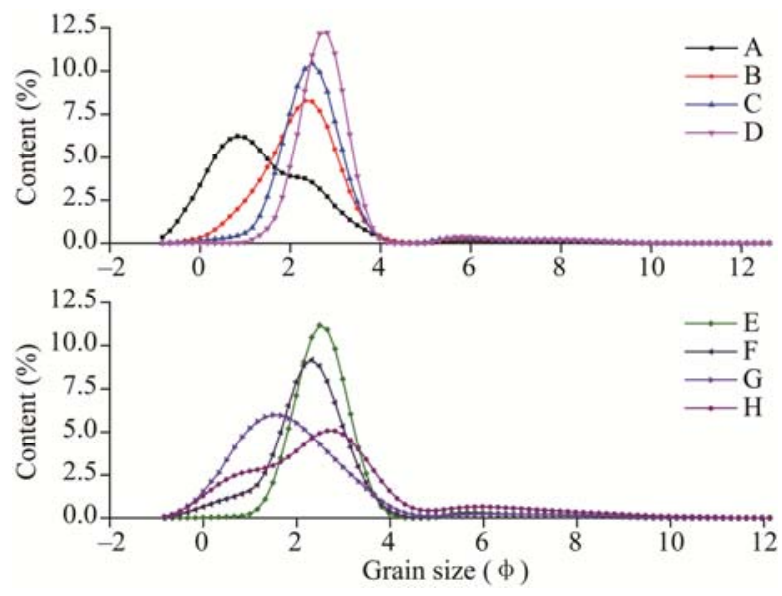

Fig. 3 Curves for the grain-size distributions at the eight sampling positions on the linear dunes and in the interdune corridors. A-H mean the positions of the samples.

platykurtic curve. The curves for sediments from the base of the SSW flank and the interdune corridor show a trimodal distribution. In addition to the two peaks mentioned above (i.e. fine sand and silt), they have a third peak for coarse sand $(0.83 \phi)$. In contrast, the main peak for sediments from the interdune corridor represents fine sands (2.32 to $2.82 \phi)$. The frequency curves clearly indicate that the linear dunes in the Qarhan Salt Lake appear to consist of a population of grains transported by saltation with a modal diameter of 2 to $3 \phi$, a population of coarse grains transported by creeping or as part of the traction load and coarser than $1.5 \phi$, and a third population of suspended particles and dust finer than $3.5 \phi$ (Visher, 1969).

\subsection{Grain-size parameters of the dune and inter- dune sediments}

Figure 4 shows the variation in the mean values of the grain-size parameters among the sampling locations on the dune and in the interdune corridor. Figures 5 and 6 show how these grain-size parameters vary along the downwind transect from sampling site 1 to site 13 and in the interdune corridors. Although the range of values for the grain-size parameters is relatively small, the distributions indicate a pattern with finer sediments at the crest of the linear dunes, with the mean grain-size becoming finer from the base to crest, accompanied by increased sorting. In addition, the mean grain-size at the dune's crest tended to become

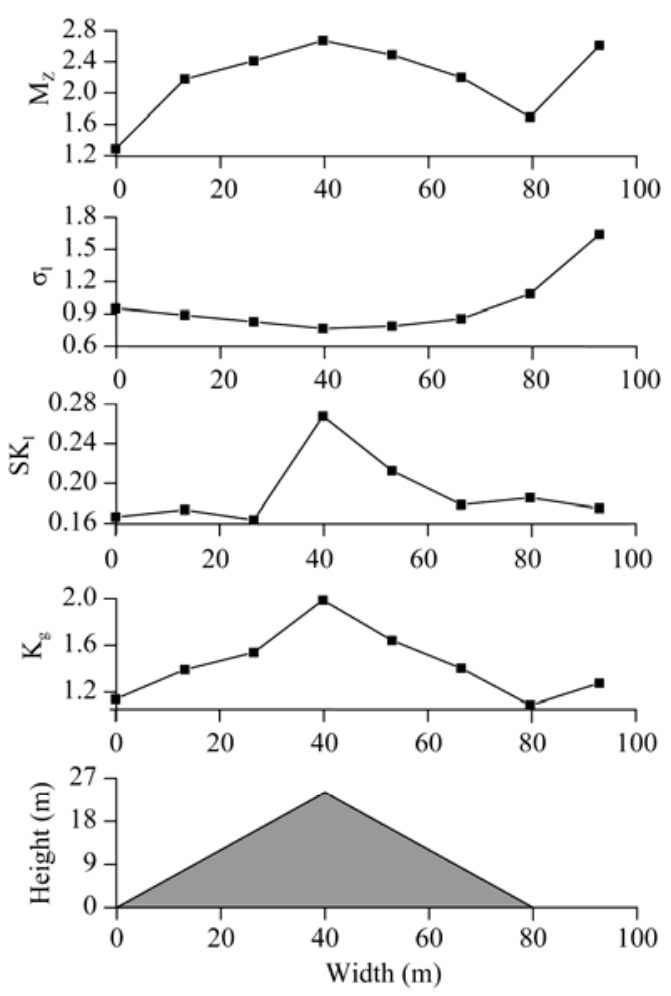

Fig. 4 Variations in the grain-size parameters among positions in the linear dune's profile, and illustration of the dune height at each position

finer along the downwind transect, although the sorting decreased. This indicates that the grain-size and sorting parameters exhibit systematic changes both for the individual dune and for the dune field as a whole.

\subsubsection{Mean grain-size of the dune}

The mean grain-size of the dune sediments ranged from 0.43 to $3.69 \phi$, with a mean of $2.13 \phi$, which indicates that the linear dunes are mainly composed of fine and medium sands. The mean grain-size tends to become finer from the dune's base (an average of 1.49 $\phi)$ to the lower flank $(2.19 \phi)$, the upper flank (2.45 $\phi)$ and the dune's crest (2.67 $\phi)$. The SSW flank has slightly coarser particles than the NNE flank, with mean grain-sizes of 1.96 and $2.12 \phi$, respectively. The mean grain-size shows a tendency to become finer along downwind transects, with a few exceptions (Fig. 5a). This pattern may result from interactions between aeolian sorting processes and the dune landforms.

Table 2 compares the mean grain-size and standard deviation (used here as a measure of sorting) for sediment samples from dune crests around the world. 


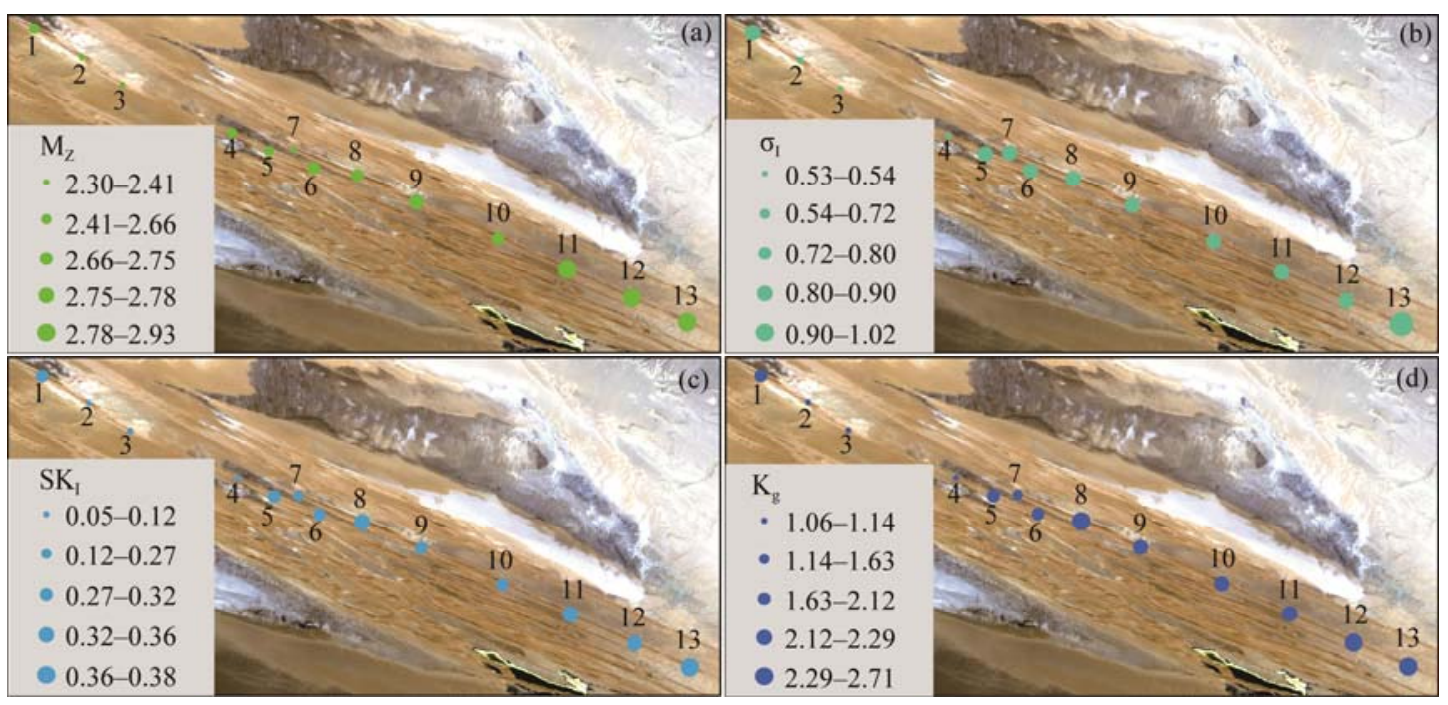

Fig. 5 Changes in the grain-size parameters of the dune crest sediments along the downwind transect

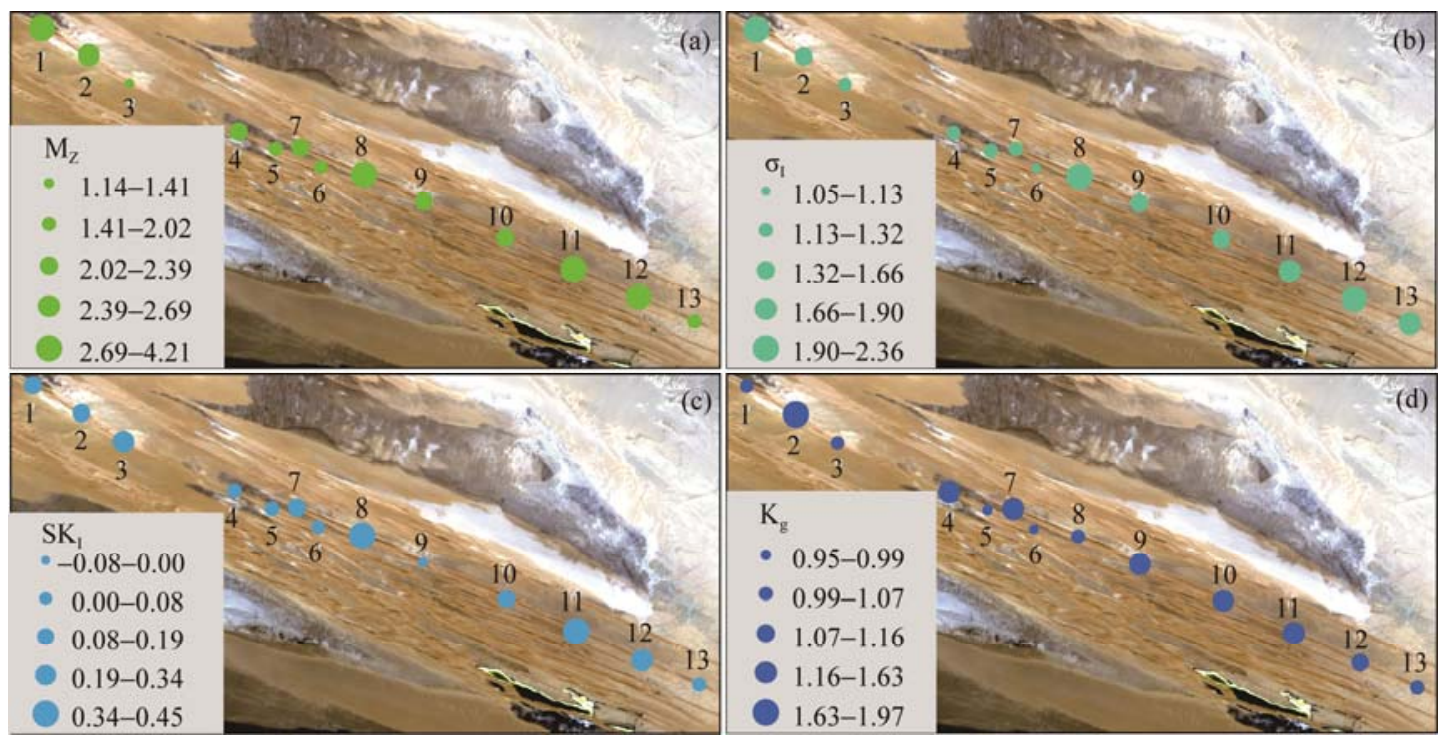

Fig. 6 Changes in the grain-size parameters of the interdune corridor sediments along the downwind transect

Table 2 A comparison of the mean grain-size $\left(\mathrm{M}_{2}\right)$ and sorting $\left(\sigma_{1}\right)$ of sediments from the crests of linear dunes around the world

\begin{tabular}{clcc}
\hline Continent or country & \multicolumn{1}{c}{ Location } & $\mathrm{M}_{\mathrm{Z}}(\phi)$ & $\sigma_{\mathrm{I}}(\phi)$ \\
\hline \multirow{2}{*}{ Australia } & Simpson Desert (Folk, 1971) & 2.53 & 0.43 \\
& Canning Basin (Ahlbrandt, 1979) & 2.02 & 0.53 \\
& Monkira (Ahlbrandt, 1979) & 2.34 & 0.35 \\
\multirow{3}{*}{ Africa } & Libya (Ahlbrandt, 1979) & 2.37 & 0.46 \\
& Namib Sand Sea (Lancaster, 1981) & 2.44 & 0.37 \\
& Southwestern Kalahari (Lancaster, 1986) & 2.16 & 0.49 \\
& Thar Desert (Goudie et al., 1973) & 2.65 & 0.56 \\
& Sinai Desert (Tsoar, 1978) & 1.87 & 0.43 \\
& Qarhan Salt Lake (This study) & 2.67 & 0.76 \\
\hline
\end{tabular}


Compared with the results of the present study, the dune crest sands are coarser in the Sinai Desert (Tsoar, 1978), Canning Basin (Ahlbrandt, 1979) and Southwestern Kalahari (Lancaster, 1986), and finer at India's Thar Desert (Goudie et al., 1973) and Australia's Simpson Desert (Folk, 1971).

\subsubsection{Sorting of the dune}

The linear dune sands had standard deviations $\left(\sigma_{\mathrm{I}}\right)$ ranging between 0.43 and 1.78 , with a mean of 0.88 , which covers the range from well to poorly sorted. The sorting increased from the dune's base $\left(\sigma_{\mathrm{I}}, 0.59\right.$ to 1.78 , with a mean of 1.02$)$ to the crest $\left(\sigma_{\mathrm{I}}, 0.53\right.$ to 1.01 , with a mean of 0.76 ), representing a change from moderately well to poorly sorted, and from moderately well to moderately sorted, respectively. The sorting was slightly better on the SSW flank than on the NNE flank, with means of 0.89 and 0.91 , respectively. However, the sorting of dune crest sands decreased along the downwind transect (Fig. 5b).

The sorting at Qarhan Salt Lake was much weaker than that of linear dunes in other regions (Table 2). Thus, our study exhibits anomalous sorting compared with other inland aeolian sand dunes. This may be because the linear dunes formed on the dry playa bed of Qarhan Salt Lake, close to the material source area, thereby leading to deficient sorting processes. In addition, the strong salt crust at the playa surface caused by the high content of soluble salts would impede aeolian sorting processes. The trend of decreasing sorting along the downwind transect may result from the increasing clay, silt and soluble salt contents in this direction.

\subsubsection{Skewness of the dune}

Skewness $\left(\mathrm{SK}_{\mathrm{I}}\right)$ represents the symmetry of the grain-size distribution. Mean values ranged from nearly symmetric to positively skewed $\left(\mathrm{SK}_{\mathrm{I}},-0.07\right.$ to 0.59 , with a mean of 0.19 ), except for one sample with negative skewness $\left(\mathrm{SK}_{\mathrm{I}},-0.11\right)$. The distributions become more positively skewed from the dune's base to its crest, with a slightly more positively skewed distribution on the NNE flank than on the SSW flank, with mean values of 0.19 and 0.17 , respectively (Fig. 4). $\mathrm{SK}_{\mathrm{I}}$ also increased along the downwind transect (Fig. 5c).

\subsubsection{Kurtosis of the dune}

Kurtosis $\left(\mathrm{K}_{\mathrm{g}}\right)$ for the sediments of the linear dunes ranged from 0.60 to 2.70 , and averaged 1.45 , which represents a range from very platykurtic to very leptokurtic. The distribution became more leptokurtic from the dune's base to its crest (Fig. 4), which is also an evidence of increasing sorting. The dune crest's sediments tended to be leptokurtic, whereas those at the dune's base and lower flanks tended to be mesokurtic. The distribution was slightly more leptokurtic on the NNE flank than on the SSW flank, with mean values of 1.38 and 1.36 , respectively. The distribution also tended to become more leptokurtic along the downwind transect (Fig. 5d).

\subsubsection{Interdune sediments}

The interdune corridor is an important component of the dune field, and plays an important role in the formation and evolution of the linear dunes. However, the grain-size parameters of the interdune corridor don't show any regular changes along the downwind transect (Fig. 6). The mean grain-size of the interdune sediments ranged from 1.14 to $4.20 \phi$, with a mean value of $2.61 \phi$, which is much finer than the means for dune sands. This indicates a high content of fine particles in the interdune corridors. The standard deviation $\left(\sigma_{\mathrm{I}}\right)$ of the interdune sediments ranged from 1.06 to 2.36 , with a mean value of 1.64 ; this represents the range from poor to moderately poor sorting. The sorting is therefore weaker than the sorting of the dune's sediments, which agrees with the results from other linear dune fields. Skewness of the interdune sands ranged from -0.09 to 0.44 , with a mean value of 0.17 , which represents a range from nearly symmetrical to strongly positively skewed. The kurtosis of the interdune sediments ranged from 0.96 to 1.96 , with a mean value of 1.27 , which represents a range from mesokurtic to very leptokurtic.

\subsection{Relationships among the grain-size parame- ters}

Figure 7 summarizes the relationships among the grain-size parameters for the sediments of the linear dune and interdune corridor. There were few clear relationships between the pairs of grain-size parameters. However, this comparison clearly shows that the dune's 
crest and upper flanks are composed of better sorted, positively to strongly positively skewed, fine sands, whereas the dune bases are composed of less well sorted, nearly symmetrical to positively skewed, medium to coarse sands. There also seems to be a positive relationship between mean grain-size and kurtosis, with finer sands at the dune's crest and upper flanks, tending to be very leptokurtic, and with coarser sands at the bases of the dune's flanks, tending to be leptokurtic or even mesokurtic. The grain-size characteristics suggest a transition between the base and upper positions on the dune. The distribution of the grain-size parameters within the linear dune's profile show considerable overlap, suggesting that the change is gradual and does not reveal clearly distinct populations of different particle sizes. In addition, the grain-size parameters of sediments from the interdune corridor show a wider range and more scattering than those of the dunes.
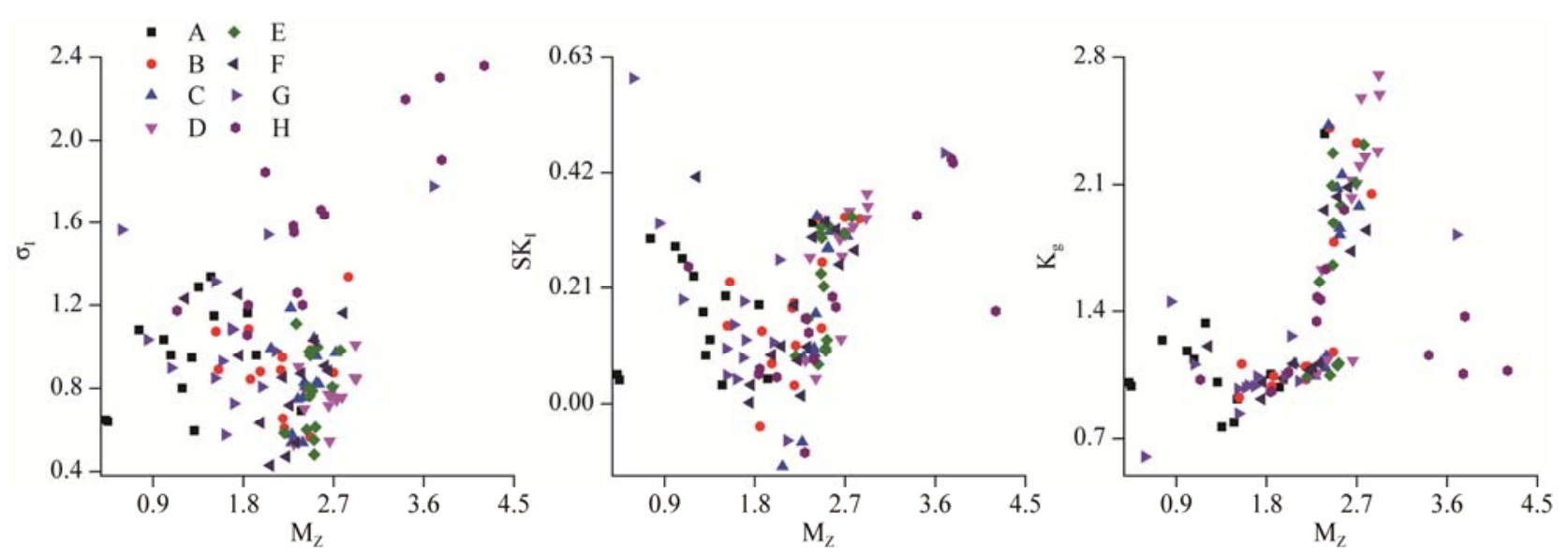

Fig. 7 The relationships between mean grain-size $\left(\mathrm{M}_{\mathrm{Z}}\right)$ and the other three parameters of the size distribution: sorting $\left(\sigma_{\mathrm{I}}\right)$, skewness $\left(\mathrm{SK}_{\mathrm{I}}\right)$ and kurtosis $\left(\mathrm{K}_{\mathrm{g}}\right)$. A-H mean the positions of the samples.

\section{Discussion}

Our data clearly show that linear dunes in the Qarhan Salt Lake region follow the pattern of finer crest sediments at the crest, with increased sorting from the dune's base to its crest. Researchers have proposed various mechanisms to account for this pattern. Bagnold (1941) attributed the coarser dune base to sorting and rolling of coarser grains along the dune's flanks under the influence of gravity and the oscillating movement of the dune's crest. Folk (1971) suggested that dune sands were selectively removed from the interdune corridors and that the grain-size difference mainly represented the combination of a similar median size with a large standard deviation. The model proposed by Folk (1971) is difficult to apply in the present study, since the interdune areas are not the source of the dune sands but rather a corridor for aeolian transport of the sands. Lancaster (1981) believed that the aeolian sorting processes that operate on linear dunes were responsible for the presence of two distinct group compositions for linear dune sands in the Namib Desert. In a comprehensive comparison of the grain-size characteristics of linear dune sediments in the Namib Desert, Southwestern Kalahari and Simpson Desert, Lancaster (1986) proposed a general model to explain the grain-size and sorting patterns for linear dune sediments based on differential movement through creep, saltation and suspension loads. The grain-size pattern of linear dunes in the present study can be explained by a combination of the models of Bagnold (1941) and Lancaster (1981, 1986).

During sand transport from the source area to the field of linear dunes, the saltation load moves faster than the creep load, causing the particles in the saltation load to be concentrated in the dunes and leaving the creep load in the interdune corridors. Furthermore, when particles travel along the dune's flanks toward the crest, the gradually increasing slope angle and decreasing kinetic energy of particles in the saltation and creep loads combine to separate the coarser grains 
from the wind stream, depositing these particles and leaving them to roll downhill along the dune flanks and become concentrated at lower parts of the flank and near the base of the dune. As sands climb progressively higher towards the dune's crest, the coarser grains are steadily left behind and the remaining particles become progressively finer and better sorted. In addition, the lateral migration of linear dunes in the study area, which was described by Hesp et al. (1989), would promote this process, as the coarser grains would roll downhill along the dune's flanks and concentrate at the dune's base. The combination of these processes would result in coarsening of the grains and weaker sorting towards the base of the flanks, leaving finer and better sorted sediments at the dune's crest.

By sampling in distinct geomorphological zones, several researchers have attempted to demonstrate the existence of two or three grain-size populations in linear dunes. For instance, Bagnold (1941) divided the profile of linear dunes into three parts. The first is the dune's crest, where the mean grain-size is relatively constant and where most of the newly deposited material slides down the dune's slip face. The second is a plinth zone, where sand particles are deposited entirely by accretion without loss of particles to downhill slides and where the mean grain-size is relatively constant. The third is a basal zone composed of coarser grains transported from the interdune corridors. Lancaster (1981) distinguished two discrete sediments in the linear dunes of the Namib Desert, namely "crest" and "plinth" sediments. In contrast, Besler (1983) divided the sediments of these Namib Desert dunes into "aeolian mobility" and "aeolian stability" zones. However, small-scale sampling by Livingstone (1987) in the Namib Desert indicated that the changes in the grain-size parameters throughout a dune's profile were gradual rather than discrete.

The present results contradict those of Bagnold (1941), Lancaster (1981) and Besler (1983), but are similar to the conclusions of Livingstone (1987). The progressive variation of grain-size parameters throughout the profile of the linear dunes in the Qarhan Salt Lake area can be attributed, on one hand, to the proximity of the dunes to their source area, and on the other hand, to the high content of silt and clay particles and soluble salts in the dune field (Rubin and
Hesp, 2009). The former explains the poor sorting of the dune sediments, and the latter explains the cohesiveness of the dune sediments. The soluble salts bind the silt and clay particles into a salt crust, which can prevent wind deflation of grains from the dunes and can decrease the efficiency of aeolian sorting processes.

The linear dunes showed a clear pattern, with finer particles in the crests than at other positions on the dune. Wilson (1972) proposed a causal link between grain-size and dune geometry (the dune height and wavelength), and it would be helpful to confirm the accuracy of this prediction for our study site and elsewhere in the world. Watson (1986) found a strong negative linear relationship between the median grain-size of linear dunes and the height above the interdune corridors. Thus, taller linear dunes should have finer crest sediments. However, the height of linear dunes in the Qarhan Salt Lake area ranges from 5 to $30 \mathrm{~m}$ (Zhou et al., 2012), whereas such dunes can reach heights of 50 to $150 \mathrm{~m}$ in the southern and central Namib Desert (Lancaster, 1981); despite this difference, the mean grain-size of crest sands in our study area was slightly finer than that in the Namib Desert. This suggests that the grain-size characteristics of the dune sediments were controlled more by the nature of the sediments in the source area and by the transport distance rather than by the dune height. Therefore, there appears to be no universal rule for the relationship between dune height and grain-size, and it seems that grain-size alone cannot determine dune geometry.

As is the case for other aeolian dune types, the formation of linear dunes is mainly controlled by three factors: a sufficient sediment supply, a suitable wind regime and suitable topography. We have already discussed the large quantities of sediments available as a source of materials for dune formation. Because there is little or no vegetation on the dunes and in the interdune corridors of our study area, vegetation is unlikely to affected the formation and evolution of the linear dunes. In terms of the wind regime, the linear dunes in the Qarhan Salt Lake area were formed in an environment characterized by a narrowly unimodal wind regime. The consistency of the wind direction is a key factor that determines the formation of the linear dunes, since a more complex wind regime would 
likely result in more complex dune morphology. The playa in our study is predominantly flat, so topography has little effect on the initial stages of the linear dunes' formation. Subsequently, the presence of linear dunes would create microtopography that would channel the winds in a single direction, thereby reinforcing the linear nature of the dunes.

Our results suggest that the characteristics of the sediment source are the dominant factors that control linear dune formation in the Qarhan Salt Lake area. Nearly 50 years ago, researchers such as Petrov (1976) noted the high content of soluble salts in the linear dunes of the study area. Rubin and Hesp (2009) found that crescent dunes transformed into linear dunes as sediment cohesiveness increased, and proposed a self-extending model for the formation of linear dunes. The gradual fining and decreased sorting observed along the downwind transect support such a conclusion. However, for clarifying the self-extending process of the linear dunes in the Qarhan Salt Lake, we should focus on the internal sedimentary structure of the dunes in future research.

\section{Conclusions}

The grain-size characteristics of the dune sediments showed clear variation between positions on the dunes, dune's crest, upper flanks and lower flanks dominated by fine sands and the flank's base dominated by medium and coarse sands. The interdune corridor sediments were characterized by a higher content of coarser and finer particles than in the dune sediments, with poorer sorting. Typical frequency curves for the particle-size distribution suggested that the linear dunes consisted of coarser particles in the creep load, finer particles in the saltation load and a finer suspension load. The clay and silt contents were remarkably higher than those in other linear dune fields around the world.

Our systematic analysis of the dune and interdune corridor sediments suggests that linear dunes in the Qarhan Salt Lake area follow the pattern with a finer crest, with increasingly fine particles and better sorting from the dune flank's base to its crest. Such a pattern can be attributed to the aeolian sorting processes that operate on the linear dunes. The lateral movement of the dune crests over time would strengthen this pattern. Compared with other linear dunes around the world, the mean grain-size of the crest sands was finer but the sorting was worse. This can be explained by the proximity to the source area of the sediments, the high proportion of particles in the suspension load and the high content of soluble salts.

\section{Acknowledgements}

The research was funded by the National Natural Science Foundation of China (41171010, 41130533, 41301003). We greatly appreciate the constructive comments from the anonymous reviewers.

\section{References}

Ahlbrandt T S. 1979. Textural parameters of eolian deposits. In: McKee E D. A Study of Global Sand Seas. Washington: U.S. Geological Survey Professional Paper 1052, 21-51.

Bagnold R A. 1941. The Physics of Blown Sand and Desert Dunes. New York: Methuen, 226-229.

Besler H. 1983. The response diagram: distinction between aeolian mobility and stability of sands and aeolian residuals by grain-size parameters. Zeitschrift für Geomorphologie Supplementband, 45: 287-301

Buckley R. 1989. Grain-size characteristics of linear dunes in central Australia. Journal of Arid Environments, 16(1): 23-28.

Edgett K S, Blumberg D G. 1994. Star and linear dunes on Mars. Icarus, 112(2): 448-464.

Folk R L, Ward W C. 1957. Brazos river bar: a study in the significance of grain-size parameters. Journal of Sedimentary Petrology, 27(1): 3-26.

Folk R L. 1971. Longitudinal dunes of the northwestern edge of the Simpson desert, Northern Territory, Australia, 1. Geomorphology and grain-size relationships. Sedimentology, 16(1-2): 5-54.

Goudie A S, Allchin B, Hegde K T M. 1973. The former extensions of the Great Indian Sand Desert. Geographical Journal, 139(2): 243-257.

Herrmann H J, Durán O, Parteli E J R, et al. 2008. Vegetation and induration as sand dunes stabilizators. Journal of Coastal Research, 24(6): 1357-1368.

Hesp P A, Hyde R, Hesp V, et al. 1989. Longitudinal dunes can move sideways. Earth Surface Processes and Landforms, 14(5): 447-451.

Kerr R C, Nigra J O. 1952. Eolian sand control. Bulletin of the American Association of Petroleum Geologists, 36(8): 1541-1573.

Lancaster N. 1981. Grain-size characteristics of Namib Desert linear dunes. Sedimentology, 28(1): 115-122.

Lancaster N. 1982. Linear dunes. Progress in Physical Geography, 6(4): 475-504.

Lancaster N. 1986. Grain-size characteristics of linear dunes in the southwestern Kalahari. Journal of Sedimentary Research, 56(3): 395-400.

Lancaster N. 1995. Geomorphology of Desert Dunes. London: Routledge, 59-70. 
Lancaster N. 2006. Linear dunes on Titan. Science, 312(5774): $702-703$.

Lee P, Thomas P C. 1995. Longitudinal dunes on Mars: Relation to current wind regimes. Journal of Geophysical Research, 100(E3): 5381-5395.

Livingstone I. 1987. Grain-size variation on a 'complex' linear dune in the Namib Desert. In: Frostick L, Reid I. Desert Sediments: Ancient and Modern, Special Publication-Geological Society of London. Oxford: Blackwell, 281-291.

Livingstone I, Warren A. 1996. Aeolian geomorphology: An introduction. Harlow: Longman, 116-119.

Livingstone I, Bullard J E, Wiggs G F S, et al. 1999. Grain-size variation on dunes in the Southwest Kalahari, Southern Africa. Journal of Sedimentary Research, 69(3): 546-552.

Lorenz R D, Wall S, Radebaugh J, et al. 2006. The sand seas of Titan: Cassini RADAR observations of longitudinal dunes. Science, 312(5774): 724-727.

Petrov M P. 1976. Deserts of the World. New York: Wiley.

Qian G Q, Dong Z B, Zhang Z C, et al. 2012. Granule ripples in the Kumtagh Desert, China: Morphology, grain-size and influencing factors. Sedimentology, 59(6): 1888-1901.

Qian Y B, Wu Z N, Yang H F, et al. 2009. Spatial heterogeneity for grain-size distribution of eolian sand soil on longitudinal dunes in the southern Gurbantunggut Desert. Journal of Arid Land, 1(1): 26-33.

Qian Z Y, Liu S Q. 1994. Research on aeolian sand characteristics and depositional structure of linear dunes. Journal of Desert Research,
14(3): 25-30. (in Chinese)

Rubin D M, Hesp P A. 2009. Multiple origins of linear dunes on Earth and Titan. Nature Geoscience, 2(9): 653-658.

Thomas D S G. 1988. Analysis of linear dune sediment-form relationships in the Kalahari Dune Desert. Earth Surface Processes and Landforms, 13(6): 545-553.

Tsoar H. 1978. The dynamics of longitudinal dunes: Final Technical Report. U.S. Army European Research Office.

Tsoar H. 1989. Linear dunes-forms and formation. Progress in Physical Geography, 13(4): 507-528.

Visher G S. 1969. Grain-size distributions and depositional processes. Journal of Sedimentary Petrology, 39(3): 1074-1106.

Warren A. 1971. Dunes in the Ténéré Desert. Geographical Journal, 137(4): 458-461.

Warren A. 1972. Observations on dunes and bi-modal sands in the Ténéré Desert. Sedimentology, 19(1-2): 37-44.

Watson A. 1986. Grain-size variations on a longitudinal dune and a barchan dune. Sedimentary Geology, 46(1-2): 49-66.

Wilson I G. 1972. Aeolian bedforms-their development and origins. Sedimentology, 19(3-4): 173-210.

Zhou J X, Zhu Y, Yuan C Q. 2012. Origin and lateral migration of linear dunes in the Qaidam Basin of NW China revealed by dune sediments, internal structures, and optically stimulated luminescence ages, with implications for linear dunes on Titan. Geological Society of America Bulletin, 124(7-8): 1147-1154. 